\title{
Pulmonary thromboendarterectomy in pediatric patients: Report of three cases
}

\author{
Ulaş Kumbasar ${ }^{1}$, Ebru Aypar², Tevfik Karagöz ${ }^{2}$, Metin Demircin¹, Rıza Doğan ${ }^{1}$ \\ ${ }^{1}$ Departments of Thoracic Surgery and ${ }^{2}$ Pediatric Cardiology, Hacettepe University Faculty of Medicine, Ankara, Turkey. \\ E-mail: ulaskumbasar@gmail.com \\ Received: 12th September 2017, Revised: 31st October 2017, Accepted: 28th November 2017
}

\begin{abstract}
SUMMARY: Kumbasar U, Aypar E, Karagöz T, Demircin M, Doğan R. Pulmonary thromboendarterectomy in pediatric patients: Report of three cases. Turk J Pediatr 2018; 60: 604-607.

Chronic thromboembolic pulmonary hypertension (CTEPH), which occurs due to impartial resolution of the pulmonary thrombus, is a rare type of pulmonary hypertension. However, most patients have an excellent longterm survival following pulmonary thromboendarterectomy (PTE). Pulmonary emboli and associated CTEPH is unusual in the pediatric population and is mostly reveals an underlying thrombophilic state. PTE is also recognized as the best therapeutic option in this subgroup of patients. In this case series, we report three young patients who had successfully undergone PTE due to pulmonary emboli and associated CTEPH.
\end{abstract}

Key words: pulmonary hypertension, pulmonary thromboendarterectomy, pediatric.

Chronic thromboembolic pulmonary hypertension (CTEPH) is an unusual, yet potentially curable type of pulmonary hypertension. ${ }^{1}$ It occurs in approximately $4 \%$ of patients after pulmonary embolism (PE) due to partial resolution of the thrombus. ${ }^{2}$ If untreated, survival is poor due to accompanying right heart failure. However, pulmonary thromboendarterectomy (PTE) improves cardiopulmonary hemodynamics, gas exchange and survival. Thus, PTE is recognized as an effective therapeutic option for this disease. ${ }^{3,4}$

$\mathrm{PE}$ is rare in the pediatric population with a reported incidence of 0.9 in 100,000 children per year. ${ }^{5}$ Accordingly, there are only a few reports regarding PTE in this subgroup of patients. In this report, we present three pediatric patients with CTEPH who underwent PTE at our institution.

\section{Case Reports}

Case 1

A 4-year-old girl was admitted to the Emergency Department with shortness of breath, diarrhea and dehydration. Biochemistry study revealed hypopotassemia $(\mathrm{K}=1.5 \mathrm{mEq} / \mathrm{L})$ and elevated brain natriuretic peptide level (470 pg/microliter). Routine hematological examination was normal with a platelet count of $192,000 / \mathrm{mm}^{3}$. Echocardiography showed paradoxical septal movement and tricuspid regurgitation which is moderate to high. Computed tomography scan showed thromboembolism of the left main pulmonary artery and the basal segmental arteries (Fig. 1). Surgical thromboendarterectomy was scheduled. Following median sternotomy, cardiopulmonary bypass was initiated by cannulation of the ascending aorta and bicaval cannulation. The patient was cooled to $18^{\circ} \mathrm{C}$, aorta was cross-clamped and cardiac arrest was accomplished by using cold-blood cardioplegia. Right atrium was explored via right atriotomy and the thrombus was removed. Organized thrombotic material was removed through a left pulmonary incision up to the segmental branches (Fig. 2). Mean pulmonary artery pressure dropped from 54 to $25 \mathrm{mmHg}$. The chest was closed routinely. The patient was transferred to the intensive care unit with intravenous heparin, dopamine and iloprost infusions and with stable hemodynamics. Early postoperative course was uneventful and the patient was extubated on postoperative $2^{\text {nd }}$ day. However, on postoperative $8^{\text {th }}$ day, urine output began to decline; hypotension, abdominal distension and hyperpotassemia occurred. 
The patient was evaluated and hemodialysis was planned. Although acute mesenteric occlusion was suspected, surgical treatment was not recommended by general surgeons. Abdominal distension gradually increased, low cardiac output state progressed and eventually the patient died. Mortality was thought to be associated with acute mesenteric occlusion. Post-mortem examination was not consented by the patient's family.

\section{Case 2}

A 13-year-old boy was admitted to a hospital with exertional dyspnoea and chest pain which gradually increased over the last six months. His chest CT indicated a diagnosis of bilateral pulmonary thrombus. He had a history of familial elevated factor VIII levels and hypofibrinogenemia. He was transferred to our hospital for surgical intervention. His factor VIII and fibrinogen levels were measured as $310.4 \mathrm{U} / \mathrm{dL}$ and $100 \mathrm{mg} / \mathrm{dL}$, respectively. Echocardiography revealed severely dilated right atrium (RA) and ventricle (RV), septum deviation towards the left ventricle, severe pulmonary insufficiency and an immobile, ovalshaped thrombus, within the main pulmonary artery, riding on the pulmonary bifurcation. CT scan of the thorax confirmed the thrombus in the main pulmonary trunk extending into both proximal pulmonary arteries, totally occluding the right side (Fig. 3). Bilateral pulmonary thromboendarterectomy was performed up to the segment arteries (Fig. 4). Mean pulmonary artery pressure dropped from 63 to $22 \mathrm{mmHg}$ postoperatively. An inferior vena cava (IVC) filter was inserted due to persisting right deep femoral vein thrombosis and new onset right iliac vein thrombus. At $7^{\text {th }}$ year post-operative follow-up, he was clinically well with no signs of ongoing pulmonary hypertension or recurrent emboli.

\section{Case 3}

A 10-year-old boy was admitted to hospital with dyspnoea, chest pain and syncope. His echocardiography and chest $\mathrm{CT}$ revealed bilateral pulmonary thrombus and the patient was referred to our hospital. He was evaluated for evidence of an underlying thrombophilic state and three heterozygous mutations [Methyltetrahydrofolate reductase (MTHFR) A1298C, MTHFR C677T and 4G/5G plasminogen activator inhibitor (PAI)] were detected. Echocardiography revealed severely dilated right chambers, paradoxical septal movement, severe pulmonary insufficiency and dilated pulmonary arteries with mean pulmonary artery pressure of $50 \mathrm{mmHg}$. Chest CT showed right ventricular hypertrophia, thrombus in the right main pulmonary artery involving the lobar branches with mild circumferential recanalization, filling defects in the left lingula and lower lobe artery and thrombus in the superior vena cava (SVC) (Fig. 5). Thromboendarterectomy was planned. Cardiopulmonary bypass was performed by cannulation of the ascending aorta and both vena cavas. Thrombus in the SVC was completely removed by opening the SVC proximal to the cannula. Bilateral thromboendoarterectomy was performed through a right and a left pulmonary incision under deep hypothermic circulatory arrest periods, each lasting 20 minutes (Fig. 6). Mean pulmonary artery pressure was measured as $20 \mathrm{mmHg}$. Postoperative course of the patient was uneventful.

We confirm that informed consent was taken from the families of the patients for their participations.

\section{Discussion}

$\mathrm{PE}$ and associated CTEPH is very rare in children, adolescents and young adults. 5,7 Additionally, pediatric patients make up a small portion of the CTEPH patients who have undergone PTE surgery. ${ }^{8}$ In this case series, we report three young patients who had successfully undergone PTE due to PE. As far as we know, these cases are the youngest patients who had undergone pulmonary endarterectomy in our country. CTEPH is mostly under-recognized in both adult and pediatric population. Thus, it is critical for physicians to have a high index of suspicion for the likelihood of $\mathrm{PE}$, especially in children, who have dyspnoea, chest pain and/ or syncope. Since the natural history of this disease is extremely poor without surgery and surgical treatment provides a better prognosis, we believe early and accurate diagnosis is of vital importance. ${ }^{9}$

Although specific pulmonary antihypertensive medication such as endothelin-1 antagonists, phosfodiesterase- 5 inhibitors, and prostanoids can be used, most patients experience a better hemodynamic and functional improvement and 


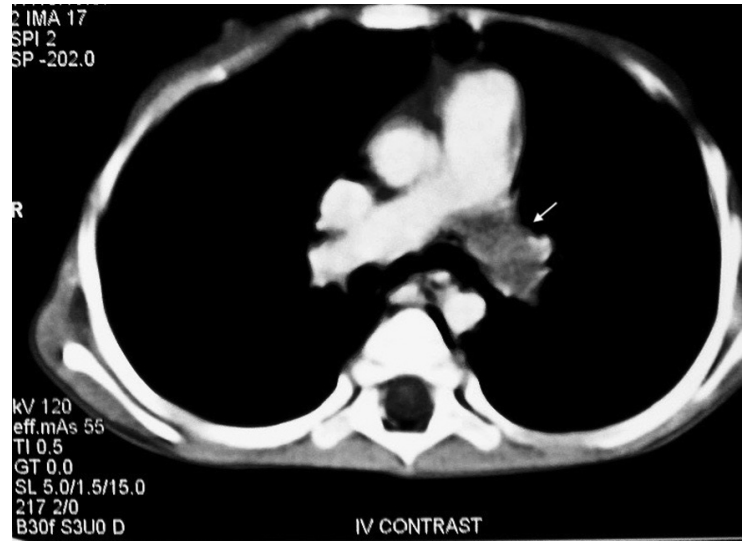

Fig. 1. CT scan showing thromboembolism of the left main pulmonary artery (white arrow).

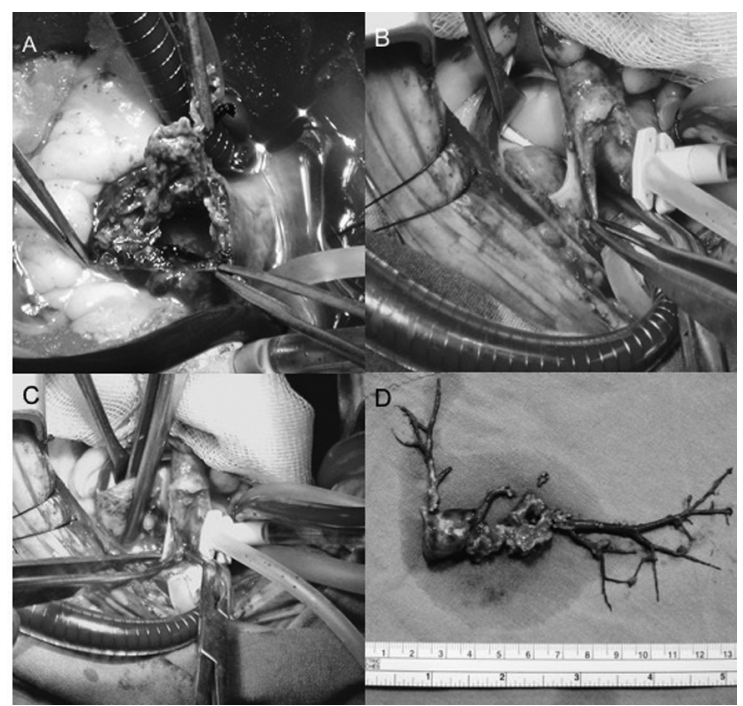

Fig. 2. A. Right atrial thrombus, B. Thrombotic material in the left main pulmonary artery, C. Removal of the thrombotic material, D. Thromboendarterectomy material up to the segmental arteries.

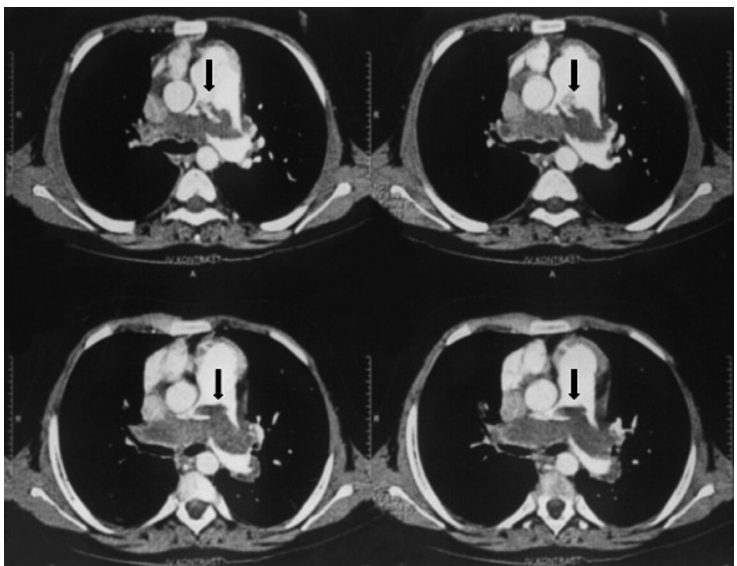

Fig. 3. CT scan showing the thrombus in the main pulmonary artery extending into both proximal pulmonary arteries (black arrows).

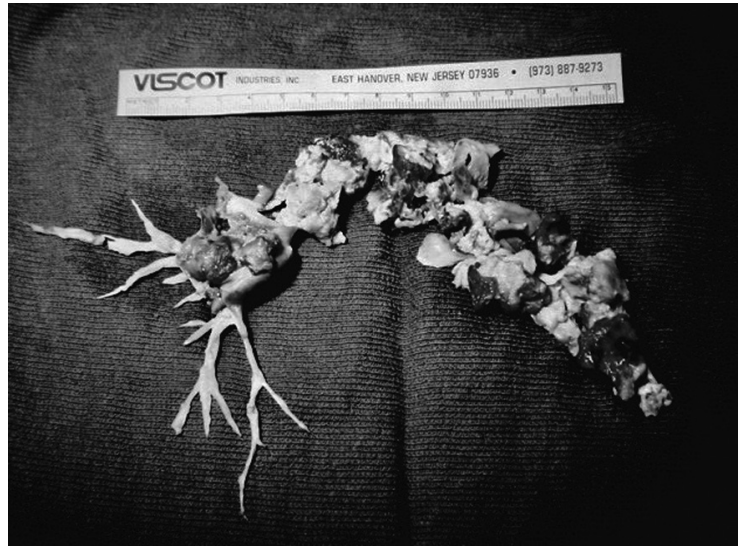

Fig. 4. Bilateral thromboendarterectomy material.

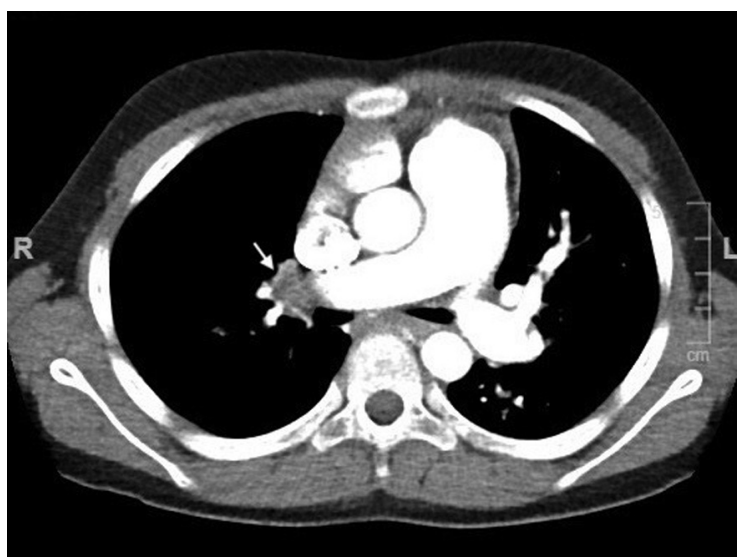

Fig. 5. CT scan showing the thrombus in the right main pulmonary artery (white arrow).

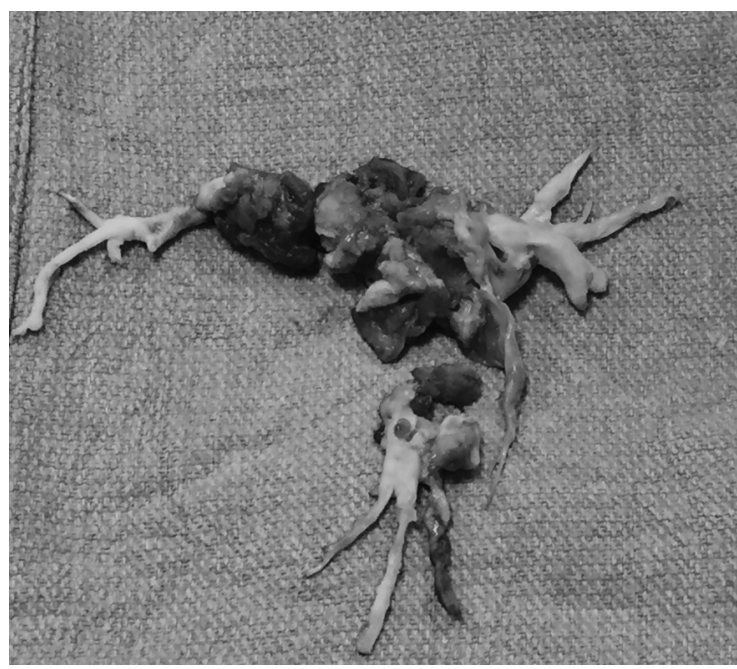

Fig. 6. Bilateral thromboendarterectomy material. 
have an excellent long-term survival following PEA $^{4,10}$. The operative steps of PEA surgery are well established for both adult and pediatric patients and include extracorporeal circulation, deep hypothermic circulatory arrest, pulmonary arteriotomy and endarterectomy up to the segmental and subsegmental branches of the pulmonary artery. ${ }^{11}$ We also performed the above-mentioned technique in all our cases and provided complete excision of the thrombotic specimen.

The youngest PTE patient was reported by Lambert et al. ${ }^{6}$ in 1999 who was a 6-month-old male with right pulmonary artery thrombus. He survived the operation and recovered well. Pediatric patients with CTEPH more often exhibited an underlying thrombophilic state compared to the adult population. Accordingly, recurrence rates are also higher in this population. Madani et al. 8,12 reported in their study that $67 \%$ of the pediatric patients with thromboembolic disease had underlying thrombophilic disorders, in contrast to the adult population in which $20 \%$ to $40 \%$ are found to have hypercoagulable state. Also, recurrent pulmonary thrombus occurred in $38 \%$ of their young patients. Although no recurrence was detected in two of our patients, two out of our three patients had underlying predisposing thrombophilic states (elevated factor VIII levels, hypofibrinogenemia and heterozygous mutations of MTHFR A1298C, C677T and 4G/5G PAI). Thus, we believe all pediatric patients with CTEPH have to be evaluated for abnormalities of blood clotting by testing for Factor V Leiden, prothrombin, MTHFR gene mutations, anti-phospholipid antibodies, protein $\mathrm{C}$, protein $\mathrm{S}$, anti-thrombin deficiencies and Factor VIII assay. Patients with hypercoagulable states should be considered for lifelong anticoagulation. The use of IVC filters in pediatric population is debatable and not advocated unless there is a risk of recurrent thrombus. ${ }^{8,12}$ We inserted a caval filter to one of our patients due to persisting femoral vein thrombus in order to avoid recurrent emboli.
In conclusion, although pediatric CTEPH is rare, successful PTE offers excellent hemodynamic and survival outcomes with low morbidity and mortality. These patients should be screened for underlying thrombophilic disorders and be followed-up closely for recurrences by a multi-disciplinary team including surgeons, hematologists, cardiologists, and rheumatologists.

\section{REFERENCES}

1. Auger WR, Fedullo FP. Chronic thromboembolic pulmonary hypertension. Semin Respir Crit Care Med 2009; 30: 471-483.

2. Thistlethwaite PA, Kaneko K, Madani MM, Jamieson SW. Technique and outcomes of pulmonary endarterectomy surgery. Ann Thorac Cardiovasc Surg 2008; 14: $274-$ 282.

3. Fedullo PF, Auger WR, Kerr KM, Rubin LJ. Chronic thromboembolic pulmonary hypertension. N Engl J Med 2001; 345: 1465-1472.

4. Archibald CJ, Auger WR, Fedullo PF, et al. Long-term outcome after pulmonary thromboendarterectomy. Am J Respir Crit Care Med 1999; 160: 523-528.

5. Stein PD, Kayali F, Olson RE. Incidence of venous thromboembolism in infants and children: Data from the National Hospital Discharge Survey. J Pediatr 2004; 145: 563-565.

6. Lambert V, Durand P, Devictor D, Planche C, Serraf A. Unilateral right pulmonary thromboendarterectomy for chronic embolism: A successful procedure in an infant. J Thorac Cardiovasc Surg 1999; 118: 953-954.

7. Bernstein D, Coupey S, Schonberg SK. Pulmonary embolism in adolescents. Am J Dis Child 1986; 140: 667-671.

8. Madani MM, Wittine LM, Auger WR, et al. Chronic thromboembolic pulmonary hypertension in pediatric patients. J Thorac Cardiovasc Surg 2011; 141: 624-630.

9. Riedel M, Stanek V, Widimsky J, Prerovsky I Longterm follow-up of patients with pulmonary thromboembolism. Late prognosis and evolution of hemodynamic and respiratory data. Chest 1982; 81: 151-158.

10. Pepke-Zaba J, Jais X, Channick R. Medical therapy in chronic thromboembolic pulmonary hypertension. Ann Am Thorac Soc 2016; 13(Suppl 3): S248-S254.

11. Kim NH, Delcroix M, Jenkins DP, et al. Chronic thromboembolic pulmonary hypertension. J Am Coll Cardiol 2013; 62(Suppl 25): D92-D99.

12. Johnson JN, Driscoll DJ, McGregor CG. Pulmonary thromboendarterectomy in adolescents and young adults. Pediatr Pulmonol 2010; 45: 614-618. 